Forecasting Marine Heatwaves using Machine Learning

Ayush Prasad, Sanxchep Sharma and Harshvardhan Agarwal

This is a non-peer reviewed preprint submitted to EarthArXiv 


\title{
Forecasting Marine Heatwaves using Machine LEARNING
}

\author{
Ayush Prasad \\ Dept. of Information Technology \\ Sikkim Manipal Institute of Technology \\ Sikkim, India \\ ayush.prd@gmail.com
}

\author{
Sanxchep Sharma \\ Dept. of Information Technology \\ Sikkim Manipal Institute of Technology \\ Sikkim, India \\ sanxchep@gmail.com
}

\author{
Harshvardhan Agarwal \\ Dept. of Computer Science \& Engineering \\ Sikkim Manipal Institute of Technology \\ Sikkim, India \\ agarwalhars10@gmail.com
}

February 1, 2022

\begin{abstract}
Recently, severe warm-water episodes have occurred frequently against a background trend of global ocean warming. Sea Surface Temperature anomalies have an impact on the integrity of marine ecosystems which is an important part of the Earth's climate system. The drastic effects of Marine Heatwaves on aquatic life have been on a steady incline in the recent years, damaging aquatic ecosystems resulting in enormous loss of marine life. The study of Marine Heatwaves has arisen as a fast-rising topic of inquiry. Operational forecasting and early warning systems that can predict such events can help in proactive planning and better mitigation strategies. In this study, the potential of machine learning models, namely Random Forest and N-BEATS, was evaluated to predict sea surface temperature on a seasonal scale using the NOAA OISST v2.1 dataset. The predicted sea surface temperature data was then used to forecast the occurrence of Marine Heatwaves up to a year in advance. The proposed models were tested across four historical Marine Heatwave events around the world. The results showed that the models were able to capture the onset, trend, and extent of the extreme events accurately.
\end{abstract}

\section{Introduction}

The rising effects of anthropogenic climate change are causing an increase in the likelihood and intensity of short-term oceanic warming events also known as Marine Heatwaves (MHWs) (Smith et al., 2021). These high-temperature extreme events have large-scale impacts on natural ecosystems and subsequent socioeconomic consequences. For e.g., the 2014-2016 MHW event in the Northeast Pacific Ocean caused a mass mortality event where 62 thousand sea birds (Uria Aalge) were found dead (Piatt et al., 2020). Similarly, the 2014-2019 MHW event in the Gulf of Alaska contributed to an economic loss of US\$ 103 million every year to the fishery industry (Barbeaux et al. 2020). A repertoire of climate resilient approaches, including improved marine heatwave forecasting, proactive resource management, and enhanced resilience, is urgently needed.

There are a limited number of studies that have tried to evaluate the potential of sea surface temperature forecasts to predict MHW. The study by (Jacox et al., 2019) is one of the earliest attempts to predict MHWs. The study tried to evaluate the occurrence of four MHW events between 2014 and 2016 in the California Current System (CCS) using 8 different coupled climate models. All the trained models were able to capture the rising temperature anomalies beginning in late 2013. The models correctly predicted warmer than average summer temperatures in 2014. During the 
second anomaly event beginning in late 2014, none of the models, including those which were trained 2 months before the event, were able to predict the rising temperatures due to the wind stress anomalies. The Authors noted that the major hurdle in using climate models for MHW prediction is that their coarse resolution makes them unable to model fine-scale ocean processes making them difficult to predict MHW events at smaller regions like the CCS.

The authors in (Benthuysen et al., 2021) investigated the potential of the Australian Community Climate and Earth System Simulator Seasonal version 1 (ACCESS-S1) ocean-atmosphere model to predict the 2020 marine heatwaves in the Great Barrier Reef on a sub-seasonal scale. The model was driven with the NOAA's daily and monthly mean interpolated outgoing longwave radiation and wind velocity, air-sea heat fluxes from the ERA5 dataset. The model was correctly able to predict the onset of MHWs a week in advance but was not able to capture the end of the MHW. The recent work by authors in (Spillman et al. 2021) also explored the use of ACCESS-S2 for developing monthly MHW forecasts, which achieved a hit rate of up to 40 percent when forecasting 4 months ahead. The authors emphasized the importance of developing more accurate seasonal forecasts.

There have been numerous studies in the past decade that have tried to predict SST, ranging from physical equationsbased ocean-climate models to recent deep learning architectures such as CNN's (Saxena, 2021), Convolutional Long Short-Term Memory (Conv-LSTM) Networks, etc. (Xiao et al., 2019) recently showed that machine learning models such as Long short-term memory (LSTM) Deep Neural Network model and AdaBoost ensemble are effective in predicting short and mid-term daily SST in the range of 1 to 10 days. (Wolff et al., 2020) is a recent study where the authors reviewed the potential of Generalised Additive Models (GAMs), Random Forest (RF), XGBoost, Multi-layer Perceptron (MLP) and Long Short Term Memory (LSTM) networks to predict the SST for 562 days. The results showed that LSTM performed poorly compared to other models as it failed to capture the high-frequency variations in the input dataset. RF performed the best among all the models, however the ensemble average of all the models showed even higher accuracy.

As demonstrated in the previous literature, forecasting SSTs accurately at a region-specific scale is the key towards accurate and reliable MHW forecasts.

In this work, the performances of mainly two data-driven models, which are Random Forests and N-BEATS, are used to forecast the occurrences of MHW events on a seasonal scale i.e., monthly forecasts. The proposed models are then tested on four historical MHW events across the world by using Hit Rate and overall model accuracy.

\section{Data and Study Areas}

In our experiments, we used SST data from the Optimum Interpolation Sea Surface Temperature (OISST) v2.1 dataset (Huang et al., 2021). OISST is an analysis dataset constructed by combining observations from different platforms (satellites, ships, buoys and Argo floats) on a 0.25 degree global grid. A spatially complete SST map is produced by interpolating to fill in the observations missing from the dataset. OISST v2.1 data are available from NOAA National Centers for Environmental Information for the time period starting from September 1, 1981 and updated daily. We used the monthly average SST values from the OISST dataset.

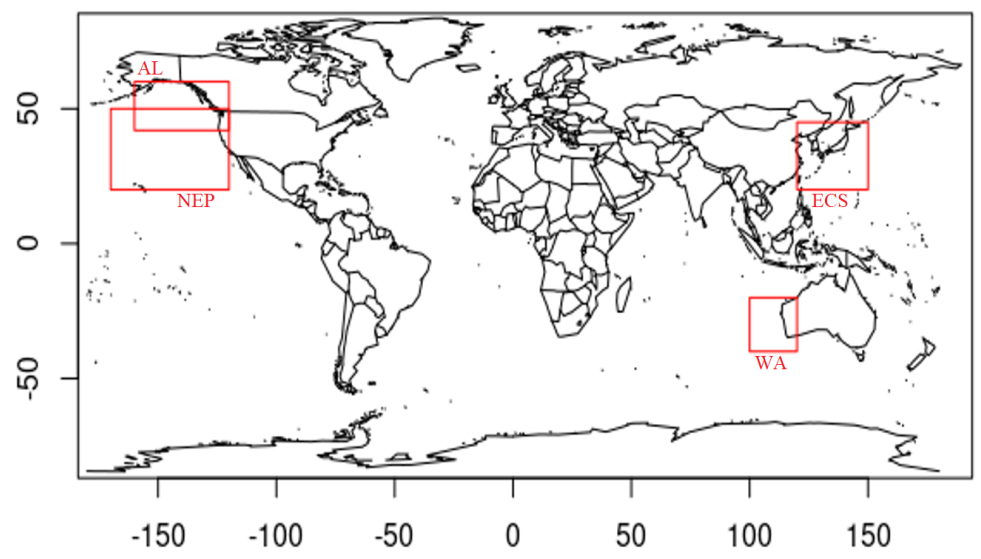

Figure 1: Study Regions where, AL - The Gulf of Alaska and the Berring Sea, NEP - Northeast Pacific, WA - West Coast of Australia, ECS - East China Seas 
Following the work by (Oliver et al. 2021), we identified four areas of interest across the world that have had intensive MHWs in the previous years (Figure 1).

It was noticed that during the Gulf of Alaska and the Berring Sea Event, the sea surface temperature increased by 1-2 degrees through September 2016 (Walsh et al., 2018). In the Northeast Pacific the temperature anomalies reached up-to $1.76^{\circ} \mathrm{C}$ throughout 2014 and 2015. On the West Coast of Australia, the SST anomalies reached as high as $5.1^{\circ} \mathrm{C}$ during the Ningaloo Niño event in 2011 (Benthuysen et al. 2014) and during East China Seas MHW event in 2016, the sea surface anomalies rose by $2^{\circ} \mathrm{C}$ (Tan and Cai, 2018).

\section{Methods}

To classify MHWs, we used the definition presented in (Scannell et al., 2020) which defines MHW as an event when SST exceeds the monthly climatological 90th percentile for at least a month using monthly data from January 1986 to December 2020. To detect MHWs in the OISST dataset we used the Python package Ocetrac (Scannell et al. 2021).

The Darts package (Herzen et al. 2021) available for Python was used to implement the below-mentioned time-series forecasting models. The training data for a study area consisted of data until the MHW event occurred. The test data was the year in which MHW events occurred. To predict the value at a time step we used previous 180 months data as lags.

\subsection{Random Forest}

Random Forest is a tree-based machine learning algorithm that consists of many individual decision trees that operate as an ensemble. Each individual tree in the RF gives a class prediction and the class with the most votes becomes the model's prediction. The reason that the RF model works well is that many uncorrelated trees will outperform any of the individual trees (Breiman, 2001), the low correlation being important as a better result of the problem statement can be achieved. To ensure that the behaviour of each individual tree is not too correlated, RF uses mainly two methods, Bagging (Bootstrap Aggregation) where decision trees are extremely sensitive to the data that they are trained on and Feature Randomness where in a decision tree, each time there is a split in the node, a feature is chosen such that it produces the most separation in the observations of the left sub-tree and the right sub-tree. In oceanography, RF has been shown to be an accurate way of modelling time series data, e.g. (Wolff et al., 2020), (Liu et al. 2015). In our experiments, we used 100 estimators and set the lags to 180 .

\subsection{N-BEATS}

Neural Basis Expansion Analysis for Interpretable Time Series or N-BEATS is a recent time series forecasting deep neural architecture. Backward and forward residual linkages, as well as a very deep stack of fully connected layers, form the foundation of the architecture. If the target output size is $L$, the time series' input data size will be an integer multiple $\mathrm{n}$ of $L$. Stacks of numerous basic Blocks, a trend, and a seasonality stack are used to process the $n * L$ dimensional input. Every basic Block starts with a four-layer fully connected stack, which is then split into two pieces, each of which connects to another set of fully connected layers. A Double (Backcast and Forecast) Residual Stacking topology is used to organize each Stack. Every subsequent Block receives an $n * L$ dimensional vector as its input, which is the result of an element-wise subtraction of the previous Block's Backcast output and input. A Stack's output consists of an $n * L$ dimensional Stack Backcast Outputs that are fed into the next Stack and an $L$ dimensional Stack Forecast Output that is element-wise summed with the relevant outputs from each Stack to generate the final L-dimensional Forecast output vector. To summarise, the overall design consists of two stacks, with the trend stack being accompanied by the seasonality stack (Oreshkin et al., 2020), as well as a double residual stacking topology mixed with the forecast-backcast principle. When comparing the performances of different univariate time-series forecasting methods, such as in financial data forecasting, N-BEATS has been found to be one of the best performing models (Karanikola et al. 2022). In our model, we used 8 layers, 12 stacks, 180 lags and trained up to 100 epochs.

\section{Results and Discussion}

\subsection{Sea Surface Temperature Forecast Evaluation}

To evaluate the SST predicted by different models, two well-known error evaluation metrics, viz. Mean Absolute Error (MAE) and Root Mean Square Error (RMSE) have been used as defined below, 


$$
\begin{gathered}
\text { RMSE }=\sqrt{\left(\frac{1}{n}\right) \sum_{i=1}^{n}\left(F_{i}-O_{i}\right)^{2}} \\
M A E=\left(\frac{1}{n}\right) \sum_{i=1}^{n}\left|F_{i}-O_{i}\right|
\end{gathered}
$$

where, $F_{i}$ is the forecasted SST Value, $O_{i}$ is the observed SST value, and $n$ is the number of coordinate points. The results are described in Table 1.

\begin{tabular}{|c|c|c|c|c|c|c|c|c|}
\hline \multirow[b]{3}{*}{ Models } & \multicolumn{8}{|c|}{ Study Area } \\
\hline & \multicolumn{2}{|c|}{$\mathrm{AL}$} & \multicolumn{2}{|c|}{ NEP } & \multicolumn{2}{|c|}{ WA } & \multicolumn{2}{|c|}{ ECS } \\
\hline & RMSE & MAE & RMSE & MAE & RMSE & MAE & RMSE & MAE \\
\hline Random Forest & 0.829 & 0.703 & 0.926 & 0.787 & 0.684 & 0.559 & 0.721 & 0.604 \\
\hline N-BEATS & 0.778 & 0.664 & 0.967 & 0.816 & 0.604 & 0.492 & 0.832 & 0.698 \\
\hline
\end{tabular}

Table 1: SST forecast evaluation results.

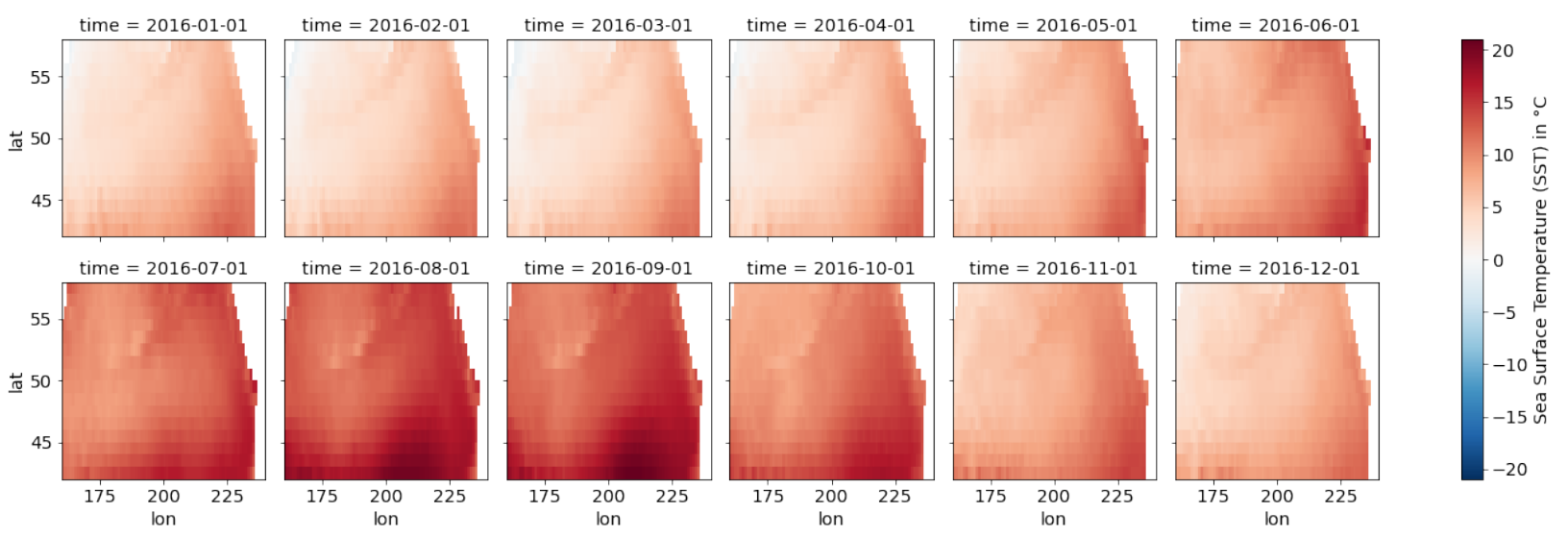

Figure 2: Observed SST in the Gulf of Alaska \& the Berring Sea for the year 2016

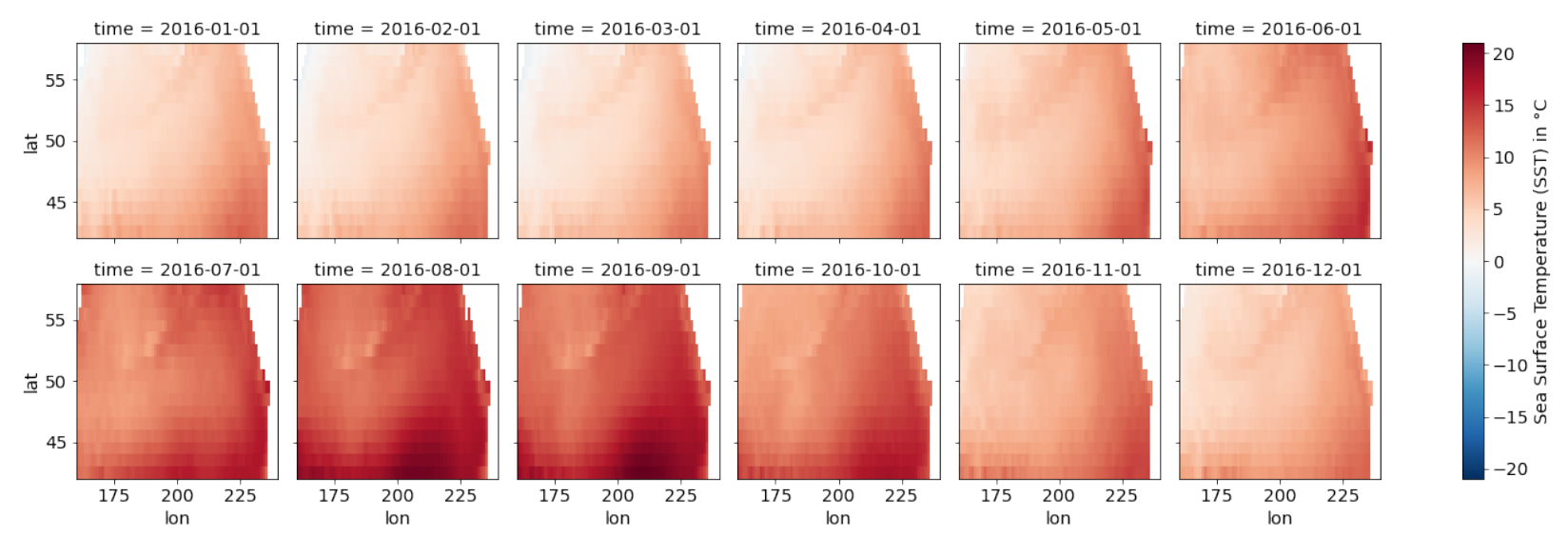

Figure 3: Forecasted SST in the Gulf of Alaska \& the Berring Sea for the year 2016 


\subsection{Marine Heatwave Forecast Evaluation}

To assess the accuracy and effectiveness of the MHW forecasts we used two metrics defined by (Benthuysen et al. 2021):

\subsubsection{Hit Rate}

Hit rate can be explained as the ratio of correctly predicted MHW events and the total number of events. The 'hits' mean that the occurrence of an MHW event has been correctly captured by the model, i.e., a true-positive heatwave prediction and 'misses' refer to a false-negative heatwave prediction. The hits and misses are first calculated for each coordinate point for a month and for a period of 12 months prediction and then the average hit rate for each study area for the entire year is calculated. The value for each hit rate for the study areas are described in Table 2.

$$
\text { Hit Rate }=\frac{\text { hits }}{\text { hits }+ \text { misses }} * 100 \%
$$
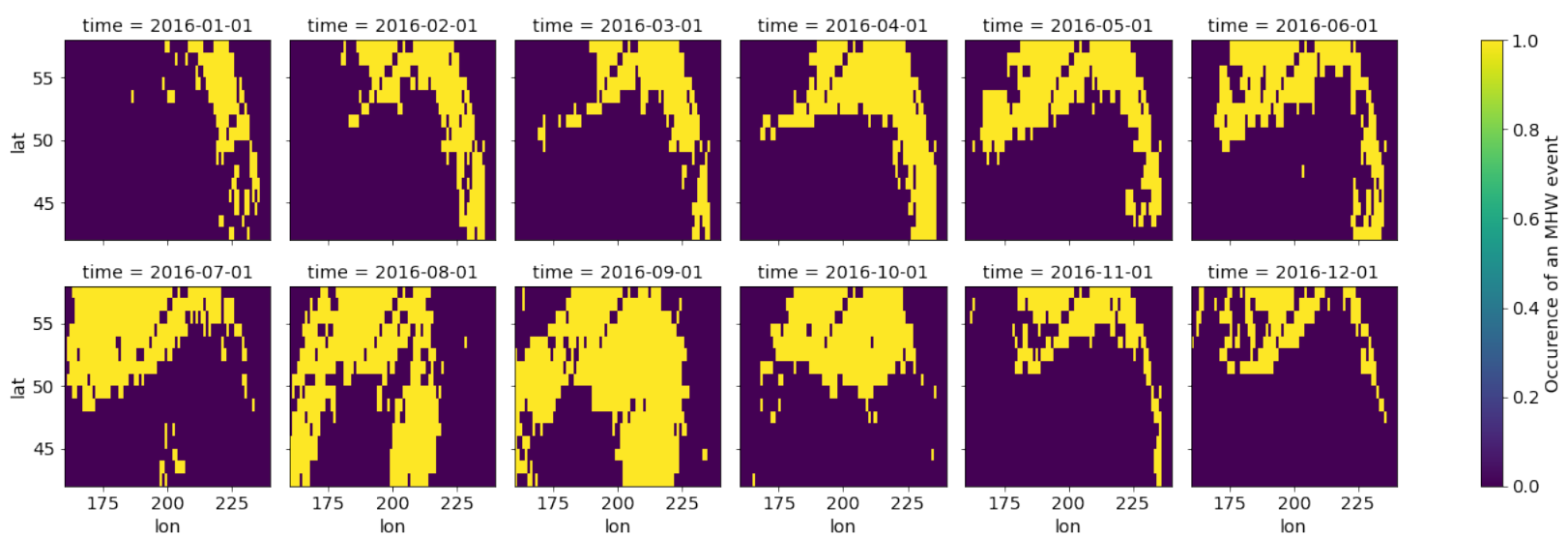

Figure 4: Observed MHW Events in the Gulf of Alaska \& the Berring Sea for the year 2016
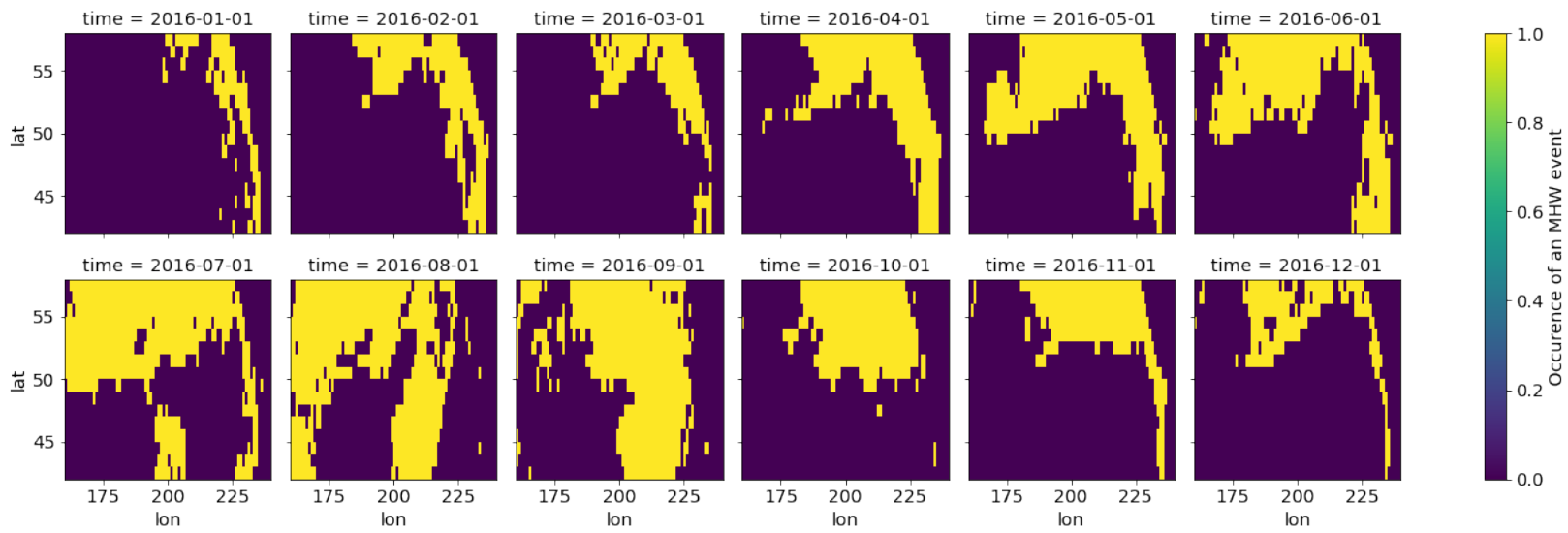

Figure 5: Forecasted MHW Events in the Gulf of Alaska \& the Berring Sea for the year 2016

\subsubsection{Accuracy}

Accuracy can be explained as the correct predictions of the model over the total coordinate points of a study area. The accuracy for each location point is calculated for each study area for a period of 12 months prediction and then the average accuracy for each study area for the entire year is calculated. The value for each accuracy for the study areas are described in Table 2. 


$$
\text { Accuracy }=\frac{\text { hits }+ \text { correct negatives }}{(\text { total number of coordinate points })} * 100 \%
$$

Table 2: Marine Heatwaves forecast evaluation results (in \%).

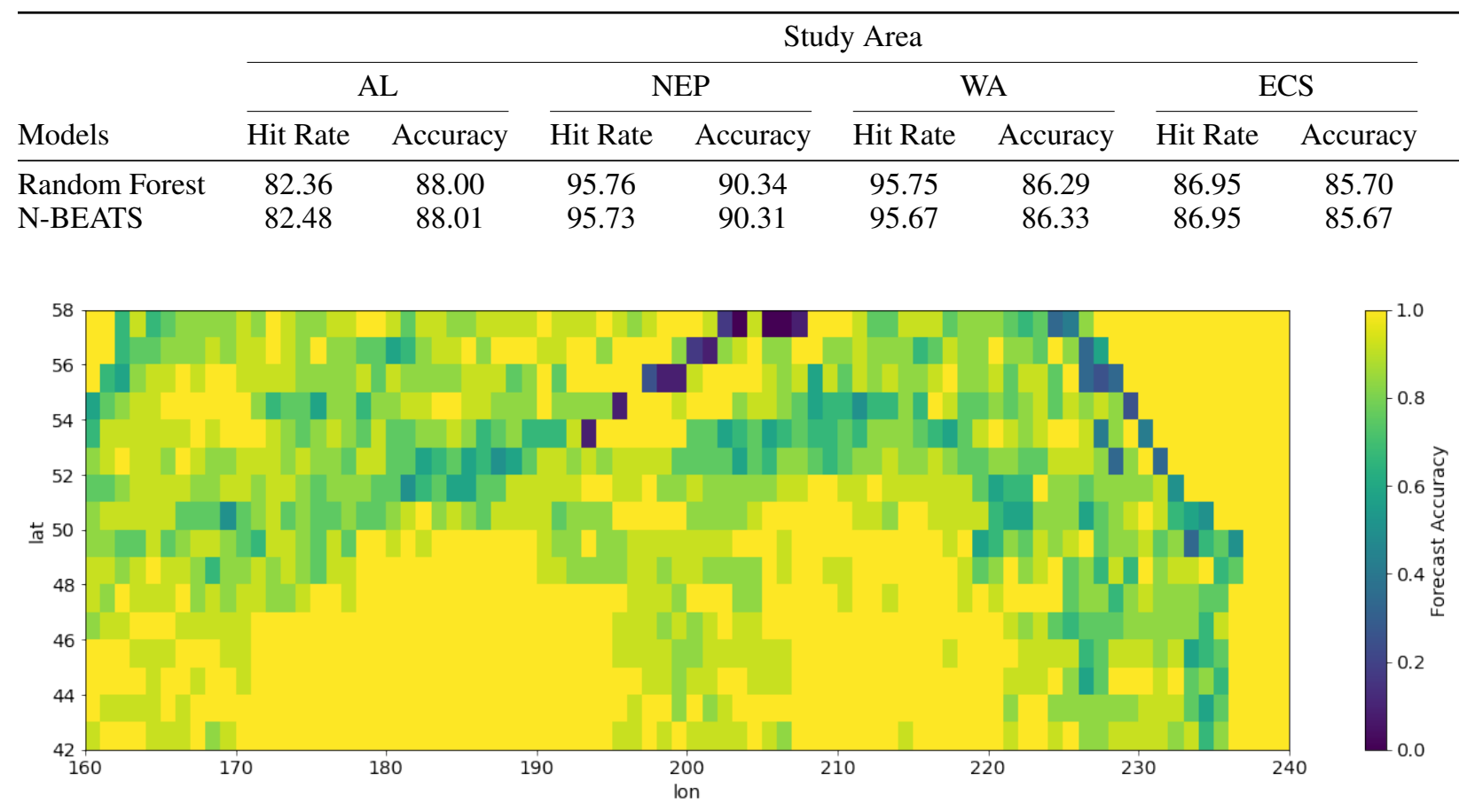

Figure 6: Accuracy density for the Gulf of Alaska and the Berring Sea for the year 2016

\subsection{Discussion}

It was noticed that both RF and N-BEATS performed similarly in predicting SST for all the four-study areas. They did not outperform each other as one of them predicted better in one study area than the other and vice-versa. Both the models were able to capture the trend, development, and extent of MHW events in all the study areas. In the Northeast

Pacific region, the SST forecasts had the highest RMSE and MAE metrics despite which the MHW forecast proved to be accurate enough to get an accuracy of above 90 percent. This could be explained by the phenomenon that the NEP region has had consistently high temperatures since 2014 (Benthuysen et al. 2014) which lowered the threshold for MHW detection. In the East China Sea, both the models achieved an accuracy of about $85 \%$ and the same hit rate of $86.5 \%$. In AL region, RF performed better in SST forecasting (Fig. 2 \& 3) and the accuracy of both the models were about $88 \%$ (Fig. $4,5 \& 6$ ). The results demonstrate that machine learning methods can be used to forecast SST and MHW events accurately with low computational cost at a seasonal scale and can be adapted to other geographical locations with varying conditions.

\section{Conclusion}

This study demonstrated the potential of machine learning methods, particularly N-BEATS and RF, for predicting MHW events on a seasonal scale. The monthly average SST values from the OISST v2.1 is used to forecast the occurrence of extreme events up to a year in advance and the proposed models were then tested across four different geographies across the world. Results showed that both the models were able to forecast the onset, extent and decline of the MHWs accurately with no significant difference in accuracy. Future improvements could include using methods like Graph Neural Networks (GNNs) to better model the spatio-temporal relations between geographical points.

Acknowledgments We thank the organizers of the 3rd NOAA Workshop on Leveraging AI in Environmental Sciences for providing us with the data used in this study and providing useful insights. 
Funding Statement This work received no specific grant from any funding agency, commercial or not-for-profit sectors.

Competing Interests The authors declare no competing interests.

Data Availability Statement All of the data and code used in this study are available in public and can be found here: https://doi.org/10.5281/zenodo.5920401

Ethical Standards The research meets all ethical guidelines, including adherence to the legal requirements of the study country.

Author Contributions Conceptualization: A.P; Methodology: A.P; S.S; H.A. Data curation: S.S. Writing original draft: A.P; S.S; H.A; All authors contributed to and approved the final submitted manuscript.

Supplementary Material No supplementary material available.

\section{References}

Steven J. Barbeaux, Kirstin Holsman, and Stephani Zador. Marine Heatwave Stress Test of Ecosystem-Based Fisheries Management in the Gulf of Alaska Pacific Cod Fishery. Frontiers in Marine Science, 7:703, August 2020. ISSN 2296-7745. doi: 10.3389/fmars.2020.00703. URL https://www.frontiersin.org/article/10.3389/fmars 2020.00703/full.

Jessica Benthuysen, Ming Feng, and Liejun Zhong. Spatial patterns of warming off Western Australia during the 2011 Ningaloo Niño: Quantifying impacts of remote and local forcing. Continental Shelf Research, 91:232-246, December 2014. ISSN 0278-4343. doi: 10.1016/j.csr.2014.09.014. URL https://www.sciencedirect.com/ science/article/pii/S0278434314003124

Jessica A. Benthuysen, Grant A. Smith, Claire M. Spillman, and Craig R. Steinberg. Subseasonal prediction of the 2020 Great Barrier Reef and Coral Sea marine heatwave. Environmental Research Letters, 16(12):124050, December 2021. ISSN 1748-9326. doi: 10.1088/1748-9326/ac3aa1. URL/https://doi.org/10.1088/1748-9326/ac3aa1. Publisher: IOP Publishing.

Leo Breiman. Random Forests. Machine Learning, 45(1):5-32, October 2001. ISSN 1573-0565. doi: 10.1023/A: 1010933404324. URL https://doi.org/10.1023/A:1010933404324

Julien Herzen, Francesco Lässig, Samuele Giuliano Piazzetta, Thomas Neuer, Léo Tafti, Guillaume Raille, Tomas Van Pottelbergh, Marek Pasieka, Andrzej Skrodzki, Nicolas Huguenin, Maxime Dumonal, Jan Kościsz, Dennis Bader, Frédérick Gusset, Mounir Benheddi, Camila Williamson, Michal Kosinski, Matej Petrik, and Gaël Grosch. Darts: User-Friendly Modern Machine Learning for Time Series. arXiv:2110.03224 [cs, stat], October 2021. URL http://arxiv.org/abs/2110.03224 arXiv: 2110.03224.

Boyin Huang, Chunying Liu, Viva Banzon, Eric Freeman, Garrett Graham, Bill Hankins, Tom Smith, and HuaiMin Zhang. Improvements of the Daily Optimum Interpolation Sea Surface Temperature (DOISST) Version 2.1. Journal of Climate, 34(8):2923-2939, April 2021. ISSN 0894-8755, 1520-0442. doi: 10.1175/JCLI-D-20-0166.1. URL https://journals.ametsoc.org/view/journals/clim/34/8/JCLI-D-20-0166.1.xml. Publisher: American Meteorological Society Section: Journal of Climate.

Michael G. Jacox, Desiree Tommasi, Michael A. Alexander, Gaelle Hervieux, and Charles A. Stock. Predicting the Evolution of the 2014-2016 California Current System Marine Heatwave From an Ensemble of Coupled Global Climate Forecasts. Frontiers in Marine Science, 6, 2019. ISSN 2296-7745. URL https://www. frontiersin. org/article/10.3389/fmars.2019.00497.

Aikaterini Karanikola, Charalampos M. Liapis, and Sotiris Kotsiantis. A Comparison of Contemporary Methods on Univariate Time Series Forecasting. In George A. Tsihrintzis, Maria Virvou, and Lakhmi C. Jain, editors, Advances in Machine Learning/Deep Learning-based Technologies: Selected Papers in Honour of Professor Nikolaos G. Bourbakis - Vol. 2, Learning and Analytics in Intelligent Systems, pages 143-168. Springer International Publishing, Cham, 2022. ISBN 978-3-030-76794-5. doi: 10.1007/978-3-030-76794-5_8. URL https://doi.org/10.1007/ 978-3-030-76794-5_8

Meiling Liu, Xiangnan Liu, Da Liu, Chao Ding, and Jiale Jiang. Multivariable integration method for estimating sea surface salinity in coastal waters from in situ data and remotely sensed data using random forest algorithm. Computers \& Geosciences, 75:44-56, February 2015. ISSN 0098-3004. doi: 10.1016/j.cageo.2014.10.016. URL https://www.sciencedirect.com/science/article/pii/S0098300414002490. 
Eric C.J. Oliver, Jessica A. Benthuysen, Sofia Darmaraki, Markus G. Donat, Alistair J. Hobday, Neil J. Holbrook, Robert W. Schlegel, and Alex Sen Gupta. Marine Heatwaves. Annual Review of Marine Science, 13(1):313342, January 2021. ISSN 1941-1405, 1941-0611. doi: 10.1146/annurev-marine-032720-095144. URL https: //www . annualreviews.org/doi/10.1146/annurev-marine-032720-095144

Boris N. Oreshkin, Dmitri Carpov, Nicolas Chapados, and Yoshua Bengio. N-BEATS: Neural basis expansion analysis for interpretable time series forecasting. arXiv:1905.10437 [cs, stat], February 2020. URL http://arxiv.org/ abs/1905.10437, arXiv: 1905.10437.

John F. Piatt, Julia K. Parrish, Heather M. Renner, Sarah K. Schoen, Timothy T. Jones, Mayumi L. Arimitsu, Kathy J. Kuletz, Barbara Bodenstein, Marisol García-Reyes, Rebecca S. Duerr, Robin M. Corcoran, Robb S. A. Kaler, Gerard J. McChesney, Richard T. Golightly, Heather A. Coletti, Robert M. Suryan, Hillary K. Burgess, Jackie Lindsey, Kirsten Lindquist, Peter M. Warzybok, Jaime Jahncke, Jan Roletto, and William J. Sydeman. Extreme mortality and reproductive failure of common murres resulting from the northeast Pacific marine heatwave of 2014-2016. PLOS ONE, 15(1):e0226087, January 2020. ISSN 1932-6203. doi: 10.1371/journal.pone.0226087. URL https://dx.plos.org/10.1371/journal.pone.0226087.

Nikita Saxena. Efficient downscaling of satellite oceanographic data with convolutional neural networks. SIGSPATIAL Special, 12(3):46-47, January 2021. doi: 10.1145/3447994.3448011. URL https://doi .org/10.1145/3447994. 3448011 .

H. A. Scannell, G. C. Johnson, L. Thompson, J. M. Lyman, and S. C. Riser. Subsurface Evolution and Persistence of Marine Heatwaves in the Northeast Pacific. Geophysical Research Letters, 47(23), December 2020. ISSN 0094-8276, 1944-8007. doi: 10.1029/2020GL090548. URL https://onlinelibrary.wiley.com/doi/10. 1029/2020GL090548

Hillary Scannell, Ryan Abernathey, Julius Busecke, David John Gagne, LuAnne Thompson, and Daniel Whitt. Ocetrac, July 2021. URL https://zenodo.org/record/5102928

Kathryn E. Smith, Michael T. Burrows, Alistair J. Hobday, Alex Sen Gupta, Pippa J. Moore, Mads Thomsen, Thomas Wernberg, and Dan A. Smale. Socioeconomic impacts of marine heatwaves: Global issues and opportunities. Science, 374(6566):eabj3593, October 2021. ISSN 0036-8075, 1095-9203. doi: 10.1126/science.abj3593. URL https://www.science.org/doi/10.1126/science.abj3593

Claire M. Spillman, Grant A. Smith, Alistair J. Hobday, and Jason R. Hartog. Onset and Decline Rates of Marine Heatwaves: Global Trends, Seasonal Forecasts and Marine Management. Frontiers in Climate, 3:801217, December 2021. ISSN 2624-9553. doi: 10.3389/fclim.2021.801217. URL https://www.frontiersin.org/articles/10. 3389/fclim.2021.801217/full.

Hongjian Tan and Rongshuo Cai. What caused the record-breaking warming in East China Seas during August 2016? Atmospheric Science Letters, 19(10):e853, October 2018. ISSN 1530261X. doi: 10.1002/asl.853. URL https://onlinelibrary.wiley.com/doi/10.1002/asl.853

John E. Walsh, Richard L. Thoman, Uma S. Bhatt, Peter A. Bieniek, Brian Brettschneider, Michael Brubaker, Seth Danielson, Rick Lader, Florence Fetterer, Kris Holderied, Katrin Iken, Andy Mahoney, Molly McCammon, and James Partain. The High Latitude Marine Heat Wave of 2016 and Its Impacts on Alaska. Bulletin of the American Meteorological Society, 99(1):S39-S43, January 2018. ISSN 0003-0007, 1520-0477. doi: 10.1175/BAMS-D-17-0105.1. URL https://journals . ametsoc.org/view/journals/bams/99/1/bams-d-17-0105.1.xml. Publisher: American Meteorological Society Section: Bulletin of the American Meteorological Society.

Stefan Wolff, Fearghal O'Donncha, and Bei Chen. Statistical and machine learning ensemble modelling to forecast sea surface temperature. Journal of Marine Systems, 208:103347, August 2020. ISSN 0924-7963. doi: 10.1016/j.jmarsys. 2020.103347. URL https://wWw.sciencedirect.com/science/article/pii/S0924796320300439.

Changjiang Xiao, Nengcheng Chen, Chuli Hu, Ke Wang, Jianya Gong, and Zeqiang Chen. Short and mid-term sea surface temperature prediction using time-series satellite data and LSTM-AdaBoost combination approach. Remote Sensing of Environment, 233:111358, November 2019. ISSN 0034-4257. doi: 10.1016/j.rse.2019.111358. URL https://www.sciencedirect.com/science/article/pii/S0034425719303773. 